\title{
Distributed Event-Based Control Strategies for Interconnected Linear Systems
}

\author{
M. Guinaldo, D. V. Dimarogonas, K. H. Johansson, \\ J. Sánchez, S. Dormido*
}

January 9, 2013

\begin{abstract}
This paper presents a distributed event-based control strategy for a networked dynamical system consisting of $N$ linear time-invariant interconnected subsystems. Each subsystem broadcasts its state over the network according to certain triggering rules which depend on local information only. The system can converge asymptotically to the equilibrium point under the proposed control design, and the existence of a lower bound for the broadcasting period is guaranteed. A novel model-based approach is derived to reduce the communication between the agents. Simulation results show the effectiveness of the proposed approaches and illustrate the theoretical results.
\end{abstract}

\section{Introduction}

Event-based methods in control systems are intended to reduce the communication between the sensors, the controller, and the actuators in a control

${ }^{*}$ M. Guinaldo, J. Sánchez and S. Dormido are with the Department of Computer Science and Automatic Control, Spanish University of Distance Education (UNED), Spain mguinaldo, jsanchez, sdormido@dia. uned.es. D. V. Dimarogonas and K. H. Johansson are with the School of Electrical Engineering, Royal Institute of Technology (KTH), Sweden dimos, kallej@kth.se. The work of first, fourth and fifth authors was supported in part by Spanish Ministry of Economy and competitiveness under Projects DPI2007-61068 and DPI2011-27818-C02-02. The work of the second and third author was supported by the Swedish Research Council (VR), the Swedish Governmental Agency for Innovation Systems (VINNOVA) and the Swedish Foundation for Strategic Research $(\mathrm{SSF})$. 
loop. They invoke a communication between these components when a certain condition in the state is violated [1. This policy is in contrast with the time-driven control approach, in which sampling, control and actuation take place at equidistant instants of time. Recently, event-triggered control has been proposed in Networked Control Systems (NCS) for allowing a more efficient usage of the limited bandwidth of the network [2]- $[6]$.

Large-scale systems are traditionally characterized by a large number of variables and uncertainties. They are usually decomposed into smaller and more manageable subsystems to better understand and cope with them. The centralized control of large-scale systems in a networked environment would require a very accurate knowledge of the interaction between these subsystems and the consumption of a lot of computation and network resources. Hence, there is a natural interest in applying event-triggering to decentralized NCS.

There are some recent contributions on distributed event-triggered control [7]-[13]. The basic idea is that each subsystem (also called agent or node) decides when to transmit the measurements based only on local information. In the most common implementations, an event is triggered when the error of the system exceeds a tolerable bound. How this error and this bound are defined separates the different approaches in the literature, e. g. deadband control [4], Lyapunov approaches to event-based control [2, 14, 15] or selftriggered control [16, 17].

A distributed event-triggered control has been proposed in [7, 12] restricted to multi-agent system and average consensus problems. In [10] selftriggered policies are proposed to avoid the constant checking of the trigger condition. However, the control system is less robust against disturbances under these policies since these cannot be detected in the inter-event times. In [13] a decentralized control for large-scale systems is proposed under the assumption of week coupling. The design of the event triggering threshold is based on Lyapunov methods and it ensures input-to-state stability of nonlinear systems. However, a positive lower bound for the broadcasting period, i.e., the difference between successive broadcasting times, may not be achievable when the system approaches the origin. This might cause severe problems since it would require the detection of events and transmission of data infinitely fast due to possible Zeno behaviors. In a previous work of the same authors [14], the design is restricted to linear systems with perfect decoupling.

A distributed event-triggered control has also been examined in [8], in which the gains measuring the degree of interconnection satisfy a generalized smallgain condition. This design does not prevent from Zeno behavior and a variation including a constant threshold-like condition is proposed to overcome 
this issue.

The previous approaches hold a constant control input in the inter-event time. In contrast, model-based control [18] takes advantage of the knowledge of the dynamics of the system to generate a control signal based on the prediction given by the model. Few publications have exploited this idea with event-triggered sampling. An emulation approach is presented in [6] for a single loop in which a control input generator and an event generator emulate the continuous-time state feedback controller. In [12], the control signal is sampled by a first-order hold, according to the double-integrator dynamics. In [19], centralized and decentralized approaches of model-based eventtriggered control are presented. Finally, in [9], a preliminary distributed model-based design has been presented for perfect decoupled interconnected linear systems.

In this paper we present a distributed event-based control for interconnected linear systems with no perfect decoupling, in contrast to [9] in which the control design perfectly decouples the neigboring nodes. The neighboring relationship is defined in the sense of dynamical interaction between the subsystems, and it is not required to be symmetric, in contrast to [14, [19].

Furthermore, we propose a novel trigger mechanism with time-dependent trigger functions for interconnected linear systems, which has been proposed for consensus control in [12]. If the parameters of these trigger functions are adequately selected, the system presents asymptotic stability to the equilibria while guaranteing a lower bound for the minimum inter-event time. With regards to [13, 14, 19, the triggering mechanism does not continuously depend on the state of the system but on the error between the current and the latest broadcasted state, which results in that the number of generated events decreases when the system is close to the equilibrium point.

Another contribution is the analysis of the inter-event times for a distributed model-based approach with model uncertainty, which is, to the best of our knowledge addressed for the first time in this paper. For instance, in the decentralized model-based approach of [19] no analysis of the inter-event times is conducted. We also prove that, when the model uncertainty fulfills a certain condition, the model-based approach gives larger minimum interevent times. The broadcasted states by the neighbors are used by the agent to generate the control action, and the error between the states estimation and the actual states are reset to zero at event times. Respect to the aforementioned work of [6], we assume that the dynamics of each subsystem are not perfectly known and we evaluate the effect of these model uncertainties. Moreover, in the design of [6] an invertibility condition is impossed to the matrix $A$ which describes the system-free dynamics. This constraint is not required here. 
The rest of the paper is organized as follows: Section 2 contains the problem statement for this work. The proposed event-based control strategy is presented in Section 3. In Section 4, a constraint for the model uncertainty is derived so that the model-based approach performs better. Numerical simulations in Section 5 show the efficiency of the proposed strategy with respect to previous results. The conclusions in Section 6 end the paper.

\section{Problem Statement}

Consider a system of $N$ linear time-invariant subsystems. The dynamics of each subsystem are given by

$$
\dot{x}_{i}(t)=A_{i} x_{i}(t)+B_{i} u_{i}(t)+\sum_{j \in N_{i}} H_{i j} x_{j}(t), \quad \forall i=1, \ldots, N
$$

where $N_{i}$ is the set of "neighbors" of subsystem $i$, i.e., set of subsystems that directly drive agent $i$ 's dynamics, and $H_{i j}$ is the interaction term between agent $i$ and agent $j$, and $H_{i j} \neq H_{j i}$ might hold. The state $x_{i}$ of the $i$ th agent has dimension $n_{i}, u_{i}$ is the $m_{i}$-dimensional local control signal of agent $i$, and $A_{i}, B_{i}$ and $H_{i j}$ are matrices of appropriate dimensions.

A digital communication network is used, and so every subsystem can only broadcast its state information to its neighbors $j \in N_{i}$ at discrete time instances. The control law is given by

$$
u_{i}(t)=K_{i} \tilde{x}_{i}(t)+\sum_{j \in N_{i}} L_{i j} \tilde{x}_{j}(t), \quad \forall i=1, \ldots, N
$$

where $K_{i}$ is the feedback gain for the nominal subsystem $i$. We assume that $A_{i}+B_{i} K_{i}$ is Hurwitz. $L_{i j}$ is a set of decoupling gains, and $\tilde{x}_{j}(t)$ is the latest state that was broadcasted by the agent $j$ at time $t$. The times at which the agent $i$ broadcasts its state generate a sequence of broadcasting times $\left\{t_{k}^{i}\right\}_{k=0}^{\infty}$, where $t_{k}^{i}<t_{k+1}^{i}$ for all $k$. Hence, (2) can be rewriten as

$$
u_{i}(t)=K_{i} x_{i}\left(t_{k}^{i}\right)+\sum_{j \in N_{i}} L_{i j} \tilde{x}_{j}(t), \quad \forall t \in\left[t_{k}^{i}, t_{k+1}^{i}\right)
$$

Let us define the error $e_{i}(t)$ between the state and the latest broadcasted state as

$$
e_{i}(t)=x_{i}\left(t_{k}^{i}\right)-x_{i}(t), \quad t \in\left[t_{k}^{i}, t_{k+1}^{i}\right) .
$$

Rewriting (1) in terms of $e_{i}(t)$ and the control law (3), we obtain

$$
\dot{x}_{i}(t)=A_{K, i} x_{i}(t)+B_{i} K_{i} e_{i}(t)+\sum_{j \in N_{i}}\left(\Delta_{i j} x_{j}(t)+B_{i} L_{i j} e_{j}(t)\right), \quad \forall i=1, \ldots, N
$$


where $A_{K, i}=A_{i}+B_{i} K_{i}$, and $\Delta_{i j}=B_{i} L_{i j}+H_{i j}$ are the coupling terms. In general, $\Delta_{i j} \neq 0$ since the interconnections between the subsystems may be not well known, there might be model uncertainties or the matrix $B_{i}$ does not have full rank. The case of perfect decoupling has already been treated in [9] and we present here a more general and realistic result.

We also define

$$
\begin{aligned}
& A_{K}=\operatorname{diag}\left(A_{K, 1}, A_{K, 2}, \ldots, A_{K, N}\right) \\
& B=\operatorname{diag}\left(B_{1}, B_{2}, \ldots, B_{N}\right) \\
& K=\left(\begin{array}{cccc}
K_{1} & L_{12} & \cdots & L_{1 N} \\
L_{21} & K_{2} & \cdots & L_{2 N} \\
\vdots & \vdots & \ddots & \vdots \\
L_{N 1} & L_{N 2} & \cdots & K_{N}
\end{array}\right) \\
& \Delta=\left(\begin{array}{cccc}
0 & \Delta_{12} & \cdots & \Delta_{1 N} \\
\Delta_{21} & 0 & \cdots & \Delta_{2 N} \\
\vdots & \vdots & \ddots & \vdots \\
\Delta_{N 1} & \Delta_{N 2} & \cdots & 0
\end{array}\right) \\
& F=A_{K}+\Delta
\end{aligned}
$$

and the stack vectors $x=\left(x_{1}^{T}, x_{2}^{T}, \ldots, x_{N}^{T}\right)^{T}$ and

$$
e=\left(e_{1}^{T}, e_{2}^{T}, \ldots, e_{N}^{T}\right)^{T}
$$

as the state and error vectors of the overall system. Note that $H_{i j}, L_{i j}, \Delta_{i j}:=$ 0 if $j \notin N_{i}$. Let also be $n=\sum_{i=1}^{N} n_{i}$ the state and error dimension.

The dynamics of the overall system are given by

$$
\dot{x}(t)=F x(t)+B K e(t) .
$$

As the broadcasted states $\tilde{x}_{i}$ remain constant between consecutive events, the error dynamics in each interval are given by

$$
\dot{e}(t)=-F x(t)-B K e(t) .
$$

\section{$3 \quad$ Event-based control strategy}

The occurrence of an event, i.e., a broadcast over the network and a control law update, is defined by trigger functions $f_{i}$ which depend on local 
information of agent $i$ only and take values in $\mathbb{R}$. The sequence of broadcasting times $t_{k}^{i}$ are determined recursively by the event trigger function as $t_{k+1}^{i}=\inf \left\{t: t>t_{k}^{i}, f_{i}(t)>0\right\}$.

Particularly, we consider trigger functions of the form

$$
f_{i}\left(e_{i}(t)\right)=\left\|e_{i}(t)\right\|-\left(c_{0}+c_{1} e^{-\alpha t}\right), \quad \alpha>0
$$

where $c_{0} \geq 0, c_{1} \geq 0$ but both parameters cannot be zero simultaneously.

The motivations of these trigger functions (14) are the following. On one hand, static trigger functions $\left(c_{1}=0\right)$ have been vastly studied in the literature, see e. g. [4, 6]. In that case, the error is bounded by $\left\|e_{i}(t)\right\| \leq c_{0} \forall t$ and $c_{0}$ determines the ultimate set in which the state of the plant is confined around the equilibrium. Large values of $c_{0}$ allow reducing the number of events but degrades the performance. On the contrary, small values of $c_{0}$ give better performance but the average inter-event time decreases considerably. On the other hand, event-triggering rules derived using Lyapunov analysis are usually of the form $\left\|e_{i}(t)\right\| \leq \alpha_{i}\left\|x_{i}(t)\right\|$. The asymptotic convergence to the equilibrium is guaranteed but a positive lower bound for the interevent time may not be guaranteed when approaching the desired equilibria ([13, 19]). In contrast, we will prove that trigger functions (14) can give good performance while decreasing the number of events and guaranteing a minimum inter-event time even if $c_{0}=0$, if the parameters are adequately selected.

The following theorem states that the system 12 with trigger functions defined as in (14) asymptotically converges to a specified region around the equilibrium point which, without loss of generality, is assumed to be $(0, \ldots, 0)^{T}$. Moreover, if $c_{0}=0$ the convergence is asymptotical to the origin. The functions (14) bound the errors $\left\|e_{i}(t)\right\| \leq c_{0}+c_{1} e^{-\alpha t}$, since an event is triggered as soon as the norm of $e_{i}(t)$ crosses the threshold $c_{0}+c_{1} e^{-\alpha t}$.

Assumption 1 . We assume that $A_{K, i}, i=1, \ldots, N$ is diagonalizable so that the Jordan form of $A_{K, i}$ is diagonal and its elements are the eigenvalues of $A_{K, i}, \lambda_{k}\left(A_{K, i}\right) k=1, \ldots, n_{i}$.

This assumption facilitates the calculations, but we argue that the extension to general Jordan blocks is achievable and provide a related analysis of this in the Appendix.

We have mentioned before that perfect decoupling is difficult to achieve in practice. However, some assumptions on $\Delta$ are required to derive the analytical results in the sequel.

Assumption 2. $F$ is assumed to be a diagonal dominant matrix [20] and $\|\Delta\|<\min \left\{\left\|A_{K}\right\|,\left|\lambda_{\max }\left(A_{K}\right)\right|\right\}$, where $\|\cdot\|$ is the induced 2-norm and 
$\lambda_{\max }\left(A_{K}\right)=\max \left\{\Re e\left(\lambda_{k}\left(A_{K}\right)\right): \forall k=1, \ldots n\right\}$. Furthermore, the Taylor series expansion of $e^{\Delta}$ can be truncated to the first order term, that is $e^{\Delta} \approx I+\Delta+O\left(\Delta^{2}\right)$.

With these assumptions, we can now state the following theorem.

Theorem 3. The state norm of the closed-loop system (12) with trigger functions of the form (14) with $\alpha<\left|\lambda_{\max }\left(A_{K}\right)\right|-\|\Delta\|$, and for all initial conditions $x(0) \in \mathbb{R}^{n}$ and $t>0$, fulfills

$$
\begin{aligned}
\|x(t)\| \leq & \kappa_{0} \beta_{0}+e^{-\left|\lambda_{\max }\left(A_{K}\right)\right| t}\left(k_{0}\|x(0)\|-\kappa_{0} \beta_{0}-\kappa_{1} \beta_{1}\right. \\
& \left.+\|\Delta\| t\left(k_{0}\|x(0)\|-\kappa_{0}-\kappa_{1}\right)\right)+e^{-\alpha t} \kappa_{1} \beta_{1} .
\end{aligned}
$$

where $k_{0}$ is a positive constant $k_{0}=\|V\|\left\|V^{-1}\right\|$ being $V$ the matrix of the eigenvectors of $A_{K}$, and

$$
\begin{aligned}
& \beta_{0}=1+\frac{\|\Delta\|}{\left|\lambda_{\max }\left(A_{K}\right)\right|}>0 \\
& \beta_{1}=1+\frac{\|\Delta\|}{\left|\lambda_{\max }\left(A_{K}\right)\right|-\alpha}>0 \\
& \kappa_{0}=k_{0} \frac{\|B K\| \sqrt{N} c_{0}}{\left|\lambda_{\max }\left(A_{K}\right)\right|} \geq 0 \\
& \kappa_{1}=k_{0} \frac{\|B K\| \sqrt{N} c_{1}}{\left|\lambda_{\max }\left(A_{K}\right)\right|-\alpha} \geq 0,
\end{aligned}
$$

with $\kappa_{0}+\kappa_{1}>0$. Furthermore, the closed-loop system does not exhibit Zenobehavior.

Proof. The analytical solution of (12) is

$$
x(t)=e^{F t} x(0)+\int_{0}^{t} e^{F(t-s)} B K e(s) d s .
$$

From Assumption 1 the matrix $A_{K}$ is diagonalizable by construction (since each is $A_{K_{i}}$ ). Then it follows that $e^{A_{K}}=V e^{D} V^{-1}$, where $D$ is the diagonal matrix that contains the eigenvalues of $A_{K}$ and $V$ is the matrix with the corresponding eigenvectors as its columns.

Thus, from 10

$$
e^{F}=e^{A_{K}+\Delta}=e^{A_{K}} e^{\Delta}=V e^{D} V^{-1} e^{\Delta} .
$$


Hence, and from Assumption 2, the norm of the state can be bounded as:

$$
\begin{aligned}
& \|x(t)\| \leq\left\|e^{\left(A_{K}+\Delta\right) t} x(0)\right\|+\int_{0}^{t}\left\|e^{\left(A_{K}+\Delta\right)(t-s)}\right\|\|B K e(s)\| d s \\
& \approx\left\|e^{A_{K} t}(I+\Delta t) x(0)\right\|+\int_{0}^{t}\left\|e^{A_{K}(t-s)}(I+\Delta(t-s))\right\|\|B K e(s)\| d s \\
& \leq\left\|e^{A_{K} t}\right\|\|I+\Delta t\|\|x(0)\|+\int_{0}^{t}\left\|e^{A_{K}(t-s)}\right\|\|I+\Delta(t-s)\|\|B K\|\|e(s)\| d s .
\end{aligned}
$$

Trigger functions (14) bound the error as $\left\|e_{i}(t)\right\| \leq c_{0}+c_{1} e^{-\alpha t}$. Thus

$$
\begin{aligned}
& \|x(t)\| \leq k_{0}\left(\int_{0}^{t} e^{-\left|\lambda_{\max }\left(A_{K}\right)\right|(t-s)}(1+\|\Delta\|(t-s))\|B K\| \sqrt{N}\left(c_{0}+c_{1} e^{-\alpha s}\right) d s\right. \\
& \left.+\|x(0)\| e^{-\left|\lambda_{\max }\left(A_{K}\right)\right| t}(1+\|\Delta\| t)\right)=k_{0}\left(\|x(0)\| e^{-\left|\lambda_{\max }\left(A_{K}\right)\right| t}(1+\|\Delta\| t)\right. \\
& +\|B K\| \sqrt{N} c_{0} \int_{0}^{t} e^{-\left|\lambda_{\max }\left(A_{K}\right)\right|(t-s)}(1+\|\Delta\|(t-s)) d s \\
& \left.+\|B K\| \sqrt{N} c_{1} e^{-\left|\lambda_{\max }\left(A_{K}\right)\right| t} \int_{0}^{t} e^{\left(\left|\lambda_{\max }\left(A_{K}\right)\right|-\alpha\right) s}(1+\|\Delta\|(t-s)) d s\right) .
\end{aligned}
$$

For the first integral term, we have

$$
\begin{aligned}
& \int_{0}^{t} e^{-\left|\lambda_{\max }\left(A_{K}\right)\right|(t-s)}(1+\|\Delta\|(t-s)) d s \\
& =\frac{1}{\left|\lambda_{\max }\left(A_{K}\right)\right|}\left(1+\frac{\|\Delta\|}{\left|\lambda_{\max }\left(A_{K}\right)\right|}-e^{-\left|\lambda_{\max }\left(A_{K}\right)\right| t}\left(1+\frac{\|\Delta\|}{\left|\lambda_{\max }\left(A_{K}\right)\right|}+\|\Delta\| t\right)\right) .
\end{aligned}
$$

Analogously, the second integral term

$$
\begin{aligned}
& e^{-\left|\lambda_{\max }\left(A_{K}\right)\right| t} \int_{0}^{t} e^{\left(\left|\lambda_{\max }\left(A_{K}\right)\right|-\alpha\right) s}(1+\|\Delta\|(t-s)) d s \\
& =\frac{e^{-\alpha t}}{\left|\lambda_{\max }\left(A_{K}\right)\right|-\alpha}\left(1+\frac{\|\Delta\|}{\left|\lambda_{\max }\left(A_{K}\right)\right|-\alpha}\right)-\frac{e^{-\left|\lambda_{\max }\left(A_{K}\right)\right| t}}{\left|\lambda_{\max }\left(A_{K}\right)\right|-\alpha}\left(1+\frac{\|\Delta\|}{\left|\lambda_{\max }\left(A_{K}\right)\right|-\alpha}+\|\Delta\| t\right)
\end{aligned}
$$


Thus

$$
\begin{aligned}
& \|x(t)\| \leq k_{0}\left[\|x(0)\| e^{-\left|\lambda_{\max }\left(A_{K}\right)\right| t}(1+\|\Delta\| t)\right. \\
& +\frac{\|B K\| \sqrt{N} c_{0}}{\left|\lambda_{\max }\left(A_{K}\right)\right|}\left(1+\frac{\|\Delta\|}{\left|\lambda_{\max }\left(A_{K}\right)\right|}-e^{-\left|\lambda_{\max }\left(A_{K}\right)\right| t}\left(1+\frac{\|\Delta\|}{\left|\lambda_{\max }\left(A_{K}\right)\right|}+\|\Delta\| t\right)\right) \\
& \left.+\frac{\|B K\| \sqrt{N} c_{1}}{\left|\lambda_{\max }\left(A_{K}\right)\right|-\alpha}\left(e^{-\alpha t}\left(1+\frac{\|\Delta\|}{\left|\lambda_{\max }\left(A_{K}\right)\right|-\alpha}\right)-e^{-\left|\lambda_{\max }\left(A_{K}\right)\right| t}\left(1+\frac{\|\Delta\|}{\left|\lambda_{\max }\left(A_{K}\right)\right|-\alpha}+\|\Delta\| t\right)\right)\right]
\end{aligned}
$$

Recalling the definitions of $\beta_{1}, \beta_{2}, \kappa_{0}$ and $\kappa_{1}$ in $(16)$ and reordering terms, it yields

$$
\begin{aligned}
\|x(t)\| \leq & \kappa_{0} \beta_{0}+e^{-\left|\lambda_{\max }\left(A_{K}\right)\right| t}\left(k_{0}\|x(0)\|-\kappa_{0} \beta_{0}-\kappa_{1} \beta_{1}\right. \\
& \left.+\|\Delta\| t\left(k_{0}\|x(0)\|-\kappa_{0}-\kappa_{1}\right)\right)+e^{-\alpha t} \kappa_{1} \beta_{1} .
\end{aligned}
$$

Moreover, the equation above can be upper bounded, by omitting the negative terms, as

$$
\|x(t)\| \leq \kappa_{0} \beta_{0}+e^{-\left|\lambda_{\max }\left(A_{K}\right)\right| t} k_{0}\|x(0)\|(1+\|\Delta\| t)+e^{-\alpha t} \beta_{1} .
$$

We next show that Zeno behavior is excluded. Let's first assume that $c_{0}, c_{1} \neq 0$, and therefore $\kappa_{0}, \kappa_{1} \neq 0$. Let us denote the last event time occurrence as $t^{*}$. Thus, $\left\|e_{i}\left(t^{*}\right)\right\|=0$, and $f_{i}\left(t^{*}\right)=-c_{0}-c_{1} e^{-\alpha t^{*}}<0$. Therefore agent $i$ cannot trigger at the same time instant. From (4) it follows that between two consecutive events we have $\dot{e}_{i}(t)=-\dot{x}_{i}(t)$. Furthermore, from definition (11) we have $\left\|e_{i}(t)\right\| \leq\|e(t)\|$, and from (13) we derive $\|\dot{e}(t)\| \leq\|F\|\|x(t)\|+\|B K\|\|e(t)\| \leq\|F\|\|x(t)\|+\|B K\| \sqrt{N}\left(c_{0}+c_{1} e^{-\alpha t^{*}}\right)$.

If the last event occurred at time $t^{*}>0$

$$
\left\|e_{i}(t)\right\| \leq\|e(t)\| \leq \int_{t^{*}}^{t}(\|F\|\|x(s)\|+\|B K\| e(s)) d s,
$$

and $\|x(t)\| \leq\left\|x\left(t^{*}\right)\right\|$ holds in (19). Thus, defining the following constants

$$
\begin{aligned}
& k_{1}=k_{0}\|x(0)\|\|F\| \\
& k_{2}=\kappa_{1} \beta_{1}\|F\|+\|B K\| \sqrt{N} c_{1}=\|B K\| \sqrt{N} c_{1}\left(1+\frac{k_{0}\|F\| \beta_{1}}{\left|\lambda_{\max }\left(A_{K}\right)\right|-\alpha}\right) \\
& k_{3}=\kappa_{0} \beta_{0}\|F\|+\|B K\| \sqrt{N} c_{0}=\|B K\| \sqrt{N} c_{0}\left(1+\frac{k_{0}\|F\| \beta_{0}}{\lambda_{\max }\left(A_{K}\right) \mid}\right),
\end{aligned}
$$


the error can be bounded as

$$
\begin{aligned}
& \left\|e_{i}(t)\right\| \leq \int_{t^{*}}^{t}\left(k_{1} e^{-\left|\lambda_{\max }\left(A_{K}\right)\right| t^{*}}(1+\|\Delta\| s)+k_{2} e^{-\alpha t^{*}}+k_{3}\right) d s \\
& =\left(k_{1} e^{-\left|\lambda_{\max }\left(A_{K}\right)\right| t^{*}}+k_{2} e^{-\alpha t^{*}}+k_{3}\right)\left(t-t^{*}\right)+\frac{k_{1}\|\Delta\| e^{-\left|\lambda_{\max }\left(A_{K}\right)\right| t^{*}}}{2}\left(t^{2}-t^{*^{2}}\right) \\
& =\left(k_{1} e^{-\left|\lambda_{\max }\left(A_{K}\right)\right| t^{*}}\left(1+\|\Delta\| t^{*}\right)+k_{2} e^{-\alpha t^{*}}+k_{3}\right) \tau+\frac{k_{1}\|\Delta\| e^{-\left|\lambda_{\max }\left(A_{K}\right)\right| t^{*}}}{2} \tau^{2} \\
& \leq\left(k_{1}+k_{2}+k_{3}\right) \tau+\frac{k_{1}\|\Delta\|}{2} \tau^{2}
\end{aligned}
$$

where $\tau=t-t^{*}$ is the inter-event time. Note that it holds that $e^{-\left|\lambda_{\max }\left(A_{K}\right)\right| t^{*}}(1$ $\left.+\|\Delta\| t^{*}\right)<1, \forall t^{*}>0$.

The next event will not be triggered before $\left\|e_{i}(t)\right\|=c_{0}+c_{1} e^{-\alpha t} \geq c_{0}$. Thus a lower bound on the inter-events time is the solution of

$$
a \tau^{2}+b \tau=c_{0}
$$

where

$$
\begin{aligned}
& a=\frac{k_{1}\|\Delta\|}{2} \\
& b=k_{1}+k_{2}+k_{3} .
\end{aligned}
$$

Since $a$ and $b$ are positive constants, only one of the solutions of 22 is strictly positive and hence feasible, and is given by

$$
\tau=\frac{-b+\sqrt{b^{2}+4 a c_{0}}}{2 a}
$$

Remark 4. Note that the integrability of $e(t)$ in $(17)$ is justified by the definition of the event-triggered functions $f_{i}\left(e_{i}(t)\right)$, which by continuity guarantee that $e_{i}(t)$ cannot be updated to zero immediately after it had done so. Thus there is an arbitrarily small, yet positive lower bound on the interexecution times. Thus the right hand side of the ODE that described the closed loop system is piecewise continuous. Note that the specific lower bounds on the interexecution times is established in the final part of the proof.

We next analyze two particular cases: static trigger functions, i.e., $c_{1}=0$, and pure exponential trigger functions, i.e., $c_{0}=0$. After that, we particularize the derived expressions to perfect decoupling with $\Delta=0$. 


\subsection{Static trigger functions}

If $c_{1}=0$ in (14), the error is bounded by $\left\|e_{i}(t)\right\| \leq c_{0}$. The analytical expressions of Theorem 3 can be adapted to this case, and hence, the state is bounded by

$$
\|x(t)\| \leq \kappa_{0} \beta_{0}+e^{-\left|\lambda_{\max }\left(A_{K}\right)\right| t}\left(k_{0}\|x(0)\|-\kappa_{0} \beta_{0}+\|\Delta\| t\left(k_{0}\|x(0)\|-\kappa_{0}\right)\right),
$$

since $\kappa_{1}=0$ (see (16)). Also, the lower bound for the inter-event time (24) is a feasible solution if $c_{1}=0$, since it yields $k_{2}=0$ but $k_{1}, k_{3}>0$ (see (201). Thus, $a$ and $b$ in (23) are strictly positive and there is always a positive solution of $\tau$ in $(24)$.

\subsection{Pure exponential trigger functions}

In the case $c_{0}=0$ the error is bounded by $\left\|e_{i}(t)\right\| \leq c_{1} e^{-\alpha t}$, and so the error goes to zero when times goes to infinity. The analysis to derive a lower bound for the inter-event time is more involved in this case. However, the state bound (15) can be particularized for pure exponential trigger functions. The bound is computed as

$\|x(t)\| \leq e^{-\left|\lambda_{\max }\left(A_{K}\right)\right| t}\left(k_{0}\|x(0)\|-\kappa_{1} \beta_{1}+\|\Delta\| t\left(k_{0}\|x(0)\|-\kappa_{1}\right)\right)+e^{-\alpha t} \kappa_{1} \beta_{1}$.

In order to prove that the Zeno behavior is excluded, we consider the bound on $\left\|e_{i}(t)\right\|$ defined in (21) before the last inequality, i.e.

$$
\left\|e_{i}(t)\right\| \leq\left(k_{1} e^{-\left|\lambda_{\max }\left(A_{K}\right)\right| t^{*}}\left(1+\|\Delta\| t^{*}\right)+k_{2} e^{-\alpha t^{*}}\right) \tau+\frac{k_{1}\|\Delta\| e^{-\left|\lambda_{\max }\left(A_{K}\right)\right| t^{*}}}{2} \tau^{2},
$$

where $k_{1}, k_{2}$ are defined in (20). Note that $k_{3}=0$ since $c_{0}=0$.

The next event is not triggered before $\left\|e_{i}(t)\right\|=c_{1} e^{-\alpha t}$. Then it yields

$$
\frac{k_{1}\|\Delta\|}{2 c_{1}} e^{\left(\alpha-\left|\lambda_{\max }\left(A_{K}\right)\right|\right) t^{*}} \tau^{2}+\left(\frac{k_{1}}{c_{1}}\left(1+\|\Delta\| t^{*}\right) e^{\left(\alpha-\left|\lambda_{\max }\left(A_{K}\right)\right|\right) t^{*}}+\frac{k_{2}}{c_{1}}\right) \tau=e^{-\alpha \tau} .
$$

The right hand side of (27) is always positive. Moreover, the left hand side is strictly positive as well if $\alpha<\left|\lambda_{\max }\left(A_{K}\right)\right|-\|\Delta\|$, and it is upper bounded by the function of $\bar{\tau} \in \mathbb{R} \xi_{1}(\bar{\tau})=\frac{k_{1}\|\Delta\|}{2 c_{1}} \bar{\tau}^{2}+\left(\frac{k_{1}}{c_{1}}+\frac{k_{2}}{c_{1}}\right) \bar{\tau}$ and lower bounded by the function $\xi_{2}(\bar{\tau})=k_{2} / c_{1} \bar{\tau}$. The solution $\tau$ of (27) can be regarded as 


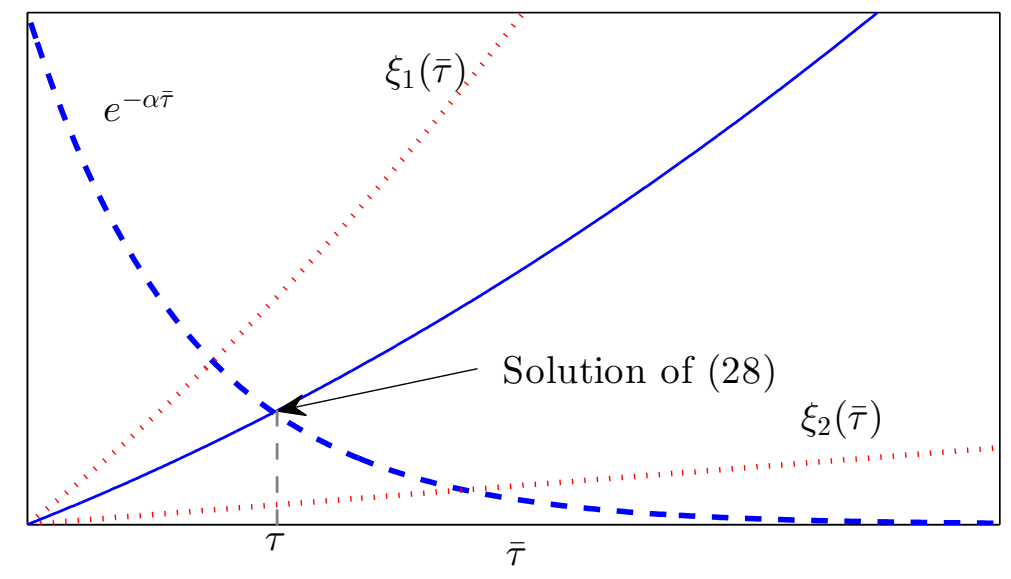

Figure 1: Graphical solution of (27).

the intersection of two real functions of $\bar{\tau}$. This solution $\bar{\tau}=\tau$ is positive $\forall t^{*} \geq 0$, and hence the lower bound for the inter-event times.

The existence of the solution $\tau$ can also be depicted graphically (see Figure 11. The solution is given by the intersection of the exponential curve and a parabola of the form $\xi(\bar{\tau})=a \bar{\tau}^{2}+b \bar{\tau}$, whose parameters $a>0$ and $b>0$ depend on $t^{*}$ and $\|\Delta\|$. Moreover, there is a single solution since the vertex coordinates of the parabola are both negative $\left(\frac{-b}{2 a}, \frac{-b^{2}}{4 a}\right)$.

\subsection{Perfect decoupling}

Perfect decoupling implies that $\Delta_{i j}=0, \forall i, j$, i.e., the decoupling gains $L_{i j}$ compensate the physical coupling between neighbors $H_{i j}$. In this case, the analysis is simplified. Specifically, the dynamics of the system are $\dot{x}(t)=$ $A_{K} x(t)+B K e(t)$ and the state $x(t)$ can be bounded for trigger functions (14) at any time $t>0$ as

$$
\|x(t)\| \leq \kappa_{0}+e^{-\left|\lambda_{\max }\left(A_{K}\right)\right| t}\left(k_{0}\|x(0)\|-\kappa_{0}-\kappa_{1}\right)+e^{-\alpha t} \kappa_{1},
$$

where $\kappa_{0}, \kappa_{1}$ have been defined in $(16)$. Note that $\beta_{0}, \beta_{1}$ are 1 .

Also, the lower bound for the inter-event time has a simpler expression than (24) since the quadratic term is zero. Thus

$$
\tau=\frac{c_{0}}{k_{1}+k_{2}+k_{3}}
$$


where the values of $k_{1}, k_{2}, k_{3}$ are also simplfied:

$$
\begin{aligned}
& k_{1}=k_{0}\|x(0)\|\|F\|=k_{0}\|x(0)\|\left\|A_{K}\right\| \\
& k_{2}=\kappa_{1} \beta_{1}\|F\|+\|B K\| \sqrt{N} c_{1}=\|B K\| \sqrt{N} c_{1}\left(1+\frac{k_{0}\left\|A_{K}\right\|}{\left|\lambda_{\max }\left(A_{K}\right)\right|-\alpha}\right) \\
& k_{3}=\kappa_{0} \beta_{0}\|F\|+\|B K\| \sqrt{N} c_{0}=\|B K\| \sqrt{N} c_{0}\left(1+\frac{k_{0}\left\|A_{K}\right\|}{\left|\lambda_{\max }\left(A_{K}\right)\right|}\right) .
\end{aligned}
$$

The details of the analysis for the perfect decoupling can also be found in [9].

\section{Model-based control}

The event-based strategy analyzed previously is based on a control law which maintains its value between two consecutive events and is based on the latest broadcasted state. One alternative to this control law can be achieved by considering that each agent has knowledge of the dynamics of its neighborhood.

In particular, let us define the control law for each agent based on a model as

$$
u_{i}(t)=K_{i} \tilde{z}_{i}(t)+\sum_{j \in N_{i}} L_{i j} \tilde{z}_{j}(t)
$$

where $\tilde{z}_{i}$ now represents the state estimation of $x_{i}$ given by the model $\left(\tilde{A}_{i}, \tilde{B}_{i}\right)$ of each isolated agent, and let us define $\tilde{A}_{K, i}=\tilde{A}_{i}+\tilde{B}_{i} K_{i}$. Let us also define $\tilde{A}_{K}=\operatorname{diag}\left(\tilde{A}_{K, 1}, \ldots, \tilde{A}_{K, n}\right)$.

The error $e_{i}(t)$ is redefined as

$$
e_{i}(t)=\tilde{z}_{i}(t)-x_{i}(t)
$$

and is reset at events' occurrence. In particular, $\tilde{z}_{i}(t)$ is computed in the inter-event times as

$$
\tilde{z}_{i}(t)=e^{\tilde{A}_{K, i}\left(t-t_{k}^{i}\right)} x_{i}\left(t_{k}^{i}\right), \quad \forall t \in\left[t_{k}^{i}, t_{k+1}^{i}\right)
$$

Note that (33) does not include the coupling effect since the decoupling gains $L_{i j}$ are designed to compensate the model of the interconnections $H_{i j}$, meaning that if $\Delta_{i j} \neq \mathbf{0}$ it is because these interconnections are partially unknown or perfect decoupling may not be possible due to, e.g., the matrix $B_{i}$ not having full rank.

Therefore, each agent $i$ has a model of its dynamics and of its neighborhood $N_{i}$. Based on this model, it estimates its state denoted as $\tilde{z}_{i}(t)$ to 


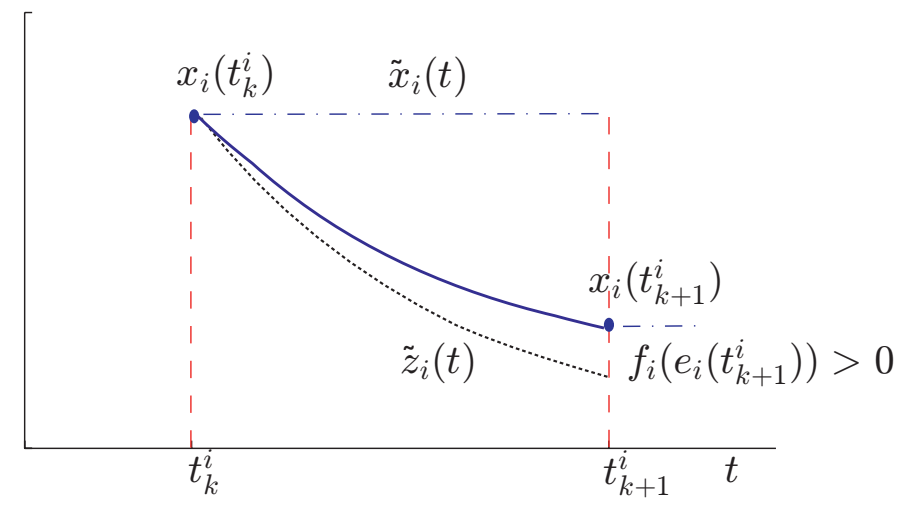

Figure 2: Model-based event-triggered control.

compute $u_{i}(t)$ in (31). When this estimation differs from a given quantity to $x_{i}(t)$, which depends on the trigger function, a new event is generated and the estimation is reset to the new measured state. For instance, $\tilde{z}_{i}$ might deviate from $x_{i}$ due to model uncertainties on $A_{K, i}$, disturbances and the effect of the non-perfect decoupling. Furthermore, the agent $i$ broadcasts the new measurement through the network to its neighbors that also update their estimations according to the new value received from agent $i$.

Figure 2 shows an example of the previous idea. In the previous sections the control law of each agent $i$ was computed based on the broadcasted measurements and it was a piecewise constant function, now we compute the control law in a model-based way between events and the error is reset at event times as we did before.

Remark 5. Note that $\tilde{z}_{i}(t)$ is used instead of $x_{i}(t)$ in the control law (31) in order to preserve the property that the agent $i$ and all its neighbors have the same version of $e_{i}(t)$. Alternatively, 12 can be redefined to deal with the aforementioned approach.

If we consider the trigger function defined in (14) and for the new error defined in (32), the state will be also bounded by (15). However, the lower bound for the inter-event time will have a different expression.

Assumption 6. Let $\bar{A}_{K}=\tilde{A}_{K}-A_{K}$ be the difference between the model and the real plant closed loop dynamics. We assume that the values of $c_{0}$ and $c_{1}$ 
satisfy the following constraint:

$$
\frac{\sqrt{N}\left(c_{0}+c_{1}\right)}{\kappa_{0} \beta_{0}+\kappa_{1} \beta_{1}}<\frac{\|F\|-\left(\left\|\bar{A}_{K}\right\|+\|\Delta\|\right)}{\left\|\tilde{A}_{K}\right\|},
$$

where $\kappa_{0}, \kappa_{1}, \beta_{0}, \beta_{1}$ have been defined in 16 .

Remark 7. Equation (34) is feasable only if the right hand side is strictly positive, since $c_{0}+c_{1}>0$. This gives a maximum value of the model uncertainty for a given bound on the norm of the coupling terms matrix or viceversa.

Theorem 8. If Assumption 6 holds, the lower bound of the broadcasting period for the system (12) when the control law for each agent is based on state estimations of the form (33), with triggering functions (14), $0<\alpha<$ $\left|\lambda_{\max }\left(A_{K}\right)\right|-\|\Delta\|$, is greater than (24).

Proof. Define the overall system state estimation as $\tilde{z}(t)=\left(\tilde{z}_{1}^{T}, \ldots, \tilde{z}_{N}^{T}\right)^{T}$. Let's prove that the bound for the inter-events time is larger in the modelbased approach. We have

$$
\begin{aligned}
\dot{e}(t) & =\dot{\tilde{z}}(t)-\dot{x}(t) \\
& =\tilde{A}_{K} \tilde{z}(t)-(F x(t)+B K e(t)) \\
& =\left(\bar{A}_{K}-\Delta\right) x(t)+\left(\tilde{A}_{K}-B K\right) e(t) .
\end{aligned}
$$

Then

$$
\begin{aligned}
\|\dot{e}(t)\| & \leq\left(\left\|\bar{A}_{K}\right\|+\|\Delta\|\right)\|x(t)\|+\left\|\tilde{A}_{K}-B K\right\|\|e(t)\| \\
& \leq\left(\left\|\bar{A}_{K}\right\|+\|\Delta\|\right)\|x(t)\|+\left\|\tilde{A}_{K}-B K\right\| \sqrt{N}\left(c_{0}+c_{1} e^{-\alpha t}\right)
\end{aligned}
$$

Assume that the last event occurred at a time $t^{*} \leq t$ and consider the case when $c_{0}, c_{1} \neq 0$. It follows that $c_{0}+c_{1} e^{-\alpha t} \leq c_{0}+c_{1} e^{-\alpha t^{*}}$. Moreover, the state norm can be bounded as in (19), thus, the error

$$
\begin{aligned}
& \left\|e_{i}(t)\right\| \leq \int_{t^{*}}^{t}\|\dot{e}(s)\| d s \leq\left(\left\|\bar{A}_{K}\right\|+\|\Delta\|\right) \frac{k_{0}\|x(0)\|\|\Delta\| e^{-\left|\lambda_{\max }\left(A_{K}\right)\right| t^{*}}}{2}\left(t^{2}-t^{* 2}\right) \\
& +\left(\left(\left\|\bar{A}_{K}\right\|+\|\Delta\|\right)\left(\kappa_{0} \beta_{0}+k_{0}\|x(0)\| e^{-\left|\lambda_{\max }\left(A_{K}\right)\right| t^{*}}+\kappa_{1} \beta_{1} e^{-\alpha t^{*}}\right)\right. \\
& \left.+\left\|\tilde{A}_{K}-B K\right\| \sqrt{N}\left(c_{0}+c_{1} e^{-\alpha t^{*}}\right)\right)\left(t-t^{*}\right)
\end{aligned}
$$

that can be rewritten as

$$
\left\|e_{i}(t)\right\| \leq\left(\tilde{k}_{1} e^{-\left|\lambda_{\max }\left(A_{K}\right)\right| t^{*}}+\tilde{k}_{2} e^{-\alpha t^{*}}+\tilde{k}_{3}\right)\left(t-t^{*}\right)+\frac{\tilde{k}_{1} e^{-\left|\lambda_{\max }\left(A_{K}\right)\right| t^{*}}\|\Delta\|\left(t^{2}-t^{* 2}\right)}{2} .
$$


As in (21), it follows that $\left\|e_{i}(t)\right\| \leq \tilde{a} \tau^{2}+\tilde{b} \tau$, where $\tau=t-t^{*}$, and

$$
\begin{aligned}
& \tilde{k}_{1}=k_{0}\|x(0)\|\left(\left\|\bar{A}_{K}\right\|+\|\Delta\|\right) \\
& \tilde{k}_{2}=\kappa_{1} \beta_{1}\left(\left\|\bar{A}_{K}\right\|+\|\Delta\|\right)+\left\|\tilde{A}_{K}-B K\right\| \sqrt{N} c_{1} \\
& \tilde{k}_{3}=\kappa_{0} \beta_{0}\left(\left\|\bar{A}_{K}\right\|+\|\Delta\|\right)+\left\|\tilde{A}_{K}-B K\right\| \sqrt{N} c_{0} \\
& \tilde{a}=\frac{\tilde{k}_{1}\|\Delta\|}{2} \\
& \tilde{b}=\tilde{k}_{1}+\tilde{k}_{2}+\tilde{k}_{3} .
\end{aligned}
$$

The next event will not occur before $\left\|e_{i}(t)\right\|=c_{0}+c_{1} e^{-\alpha t} \geq c_{0}$. This condition gives a lower bound for the broadcasting period

$$
\tau^{\prime}=\frac{-\tilde{b}+\sqrt{\tilde{b}^{2}+4 \tilde{a} c_{0}}}{2 \tilde{a}}
$$

that is larger than the lower bound in 24) if $\tilde{a}<a$ and $\tilde{b}<b$, which implies that

$$
\begin{aligned}
& \left\|\bar{A}_{K}\right\|+\|\Delta\|<\|F\| \\
& \left(\left\|\bar{A}_{K}\right\|+\|\Delta\|\right)\left(\kappa_{0} \beta_{0}+\kappa_{1} \beta_{1}\right)+\left\|\tilde{A}_{K}-B K\right\| \sqrt{N}\left(c_{0}+c_{1}\right) \\
& <\|F\|\left(\kappa_{0} \beta_{0}+\kappa_{1} \beta_{1}\right)+\|B K\| \sqrt{N}\left(c_{0}+c_{1}\right),
\end{aligned}
$$

or equivalently:

$$
\frac{\sqrt{N}\left(c_{0}+c_{1}\right)}{\kappa_{0} \beta_{0}+\kappa_{1} \beta_{1}}<\frac{\|F\|-\left(\left\|\bar{A}_{K}\right\|+\|\Delta\|\right)}{\left\|\tilde{A}_{K}-B K\right\|-\|B K\|} .
$$

The denominator on the right hand side can be bounded as:

$$
\left\|\tilde{A}_{K}-B K\right\|-\|B K\| \leq\left\|\tilde{A}_{K}\right\|+\|B K\|-\|B K\|=\left\|\tilde{A}_{K}\right\| .
$$

Then if Assumption 6 holds, (42) is fulfilled. Then, the lower bound for the broadcasting period is larger for the model-based approach.

Remark 9. Assumption 6 is not a strong assumption since when $\left\|\bar{A}_{K}\right\|$ goes to zero, $\left\|\tilde{A}_{K}\right\| \approx\left\|A_{K}\right\|$, and the right hand side of (34) can be approximated to one, since also $\|F\| \leq\left\|A_{K}\right\|+\|\Delta\|$. And for initial conditions satisfying $k_{0}\|x(0)\|>\sqrt{N}\left(c_{0}+c_{1}\right)$, it holds that

$$
c_{0}+c_{1}<\frac{\kappa_{0} \beta_{0}+\kappa_{1} \beta_{1}}{\sqrt{N}}
$$

which constraints the feseable values of $c_{0}$ and $c_{1}$. These constraints depends on the systems dynamics and the control design. 


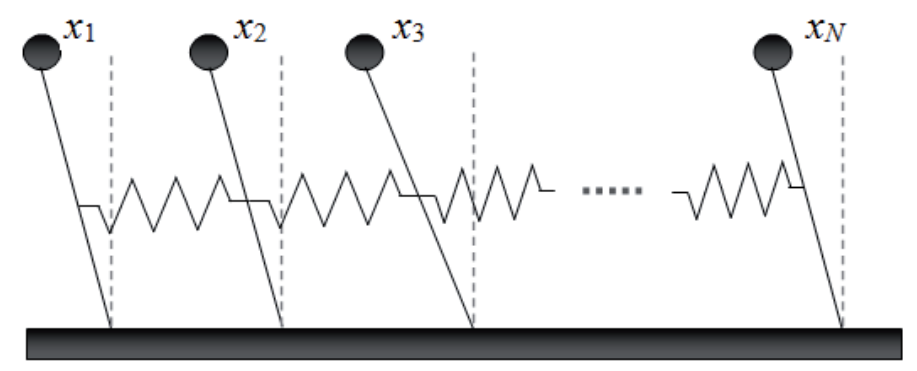

Figure 3: Scheme of the network of the inverted pendulums

\section{Simulation results}

This section presents some simulation results in order to demonstrate the effectiveness of the event-based control strategy. The system considered is a collection of $N$ inverted pendulums of mass $m$ and length $l$ coupled by springs with rate $k$ as in Figure 3. The problem of coupled oscillators has numerous applications in fields as medicine, physics or communications [21, 22], and the inverted pendulum is a well-known control engineering problem. The inverted pendulums are physically connected by springs and we desire to design control laws to reach the equilibrium as well as to decouple the system. The state of a pendulum $i$ is broadcasted to its neighbors in the chain at discrete times given by the communication strategy.

Each subsystem can be described as follows:

$$
\dot{x}_{i}(t)=\left(\begin{array}{cc}
0 & 1 \\
\frac{g}{l}-\frac{a_{i} k}{m l^{2}} & 0
\end{array}\right) x_{i}(t)+\left(\begin{array}{c}
0 \\
\frac{1}{m l^{2}}
\end{array}\right) u_{i}+\sum_{j \in N_{i}}\left(\begin{array}{cc}
0 & 0 \\
\frac{h_{i j} k}{m l^{2}} & 0
\end{array}\right) x_{j}(t)
$$

where $x_{i}(t)=\left(x_{i_{1}}(t) \quad x_{i_{2}}(t)\right)^{T}$ is the state, $a_{i}$ is the number of springs connected to the $i$ th pendulum and $h_{i j}=1, \forall j \in N_{i}$ and 0 otherwise.

State-feedback gains and decoupling gains are designed so that the system is perfectly decoupled, and each decoupled subsystem poles are at -1 and -2 . This yields the following control law:

$$
u_{i}(t)=\left(-3 m l^{2} \quad a_{i} k-\frac{m l^{2}}{4}\left(8+\frac{4 g}{l}\right)\right) \tilde{x}_{i}(t)+\sum_{j \in N_{i}}\left(\begin{array}{ll}
-k & 0
\end{array}\right) \tilde{x}_{j}(t)
$$

where $\tilde{x}_{i}(t)=\left(\begin{array}{ll}\tilde{x}_{i_{1}}(t) & \tilde{x}_{i_{2}}(t)\end{array}\right)^{T}$. In the following, the system parameters are set to $g=10, m=1, l=2$ and $k=5$. 
Table 1: Comparison of time-triggered and event-triggered strategies

\begin{tabular}{cccc} 
& No. updates & $\left\{\tau_{k}^{i}\right\}_{\min }(\mathrm{s})$ & $\left\{\tau_{k}^{i}\right\}_{\max }(\mathrm{s})$ \\
\hline \hline Time-triggered & $(28,56)$ & 0.177 & 0.3544 \\
\hline Event-triggered & 19 & 0.081 & 2.478 \\
\hline
\end{tabular}

We next present several simulation results in order to enhance the theoretical results presented previously in the paper. Furthermore, we also compare some of these results with the ones obtained in [14].

\subsection{Perfect decoupling}

The output of the system and the sequence of events for $N=4$ and initial conditions $x(0)=\left(\begin{array}{llllllll}-0.942 & 0 & 1.047 & 0 & 0.628 & 0 & -1.413 & 0\end{array}\right)^{T}$ when the trigger function is defined as in (14) with parameters $c_{0}=0.02, c_{1}=$ 0.5 and $\alpha=0.8$ is shown in Figure 4 . We consider perfect decoupling, i.e., $\Delta_{i j}=0, \forall i, j$ and control law of the form (3). The convergence of the system to a small region $\left(c_{0}=0.02\right)$ around equilibrium point is guaranteed due to the time-dependency in the trigger functions. The event generation is shown in Figure 4.b. The system converges to zero with few events. Note that the agent that generates the highest number of events is agent 2 (in red) and this value is 19 over a period of 10 seconds. Table 1 compares the proposed event-triggered approach to periodic control. The bandwidth of the closed loop subsystem is $0.8864 \mathrm{rad} / \mathrm{s}$ and the sampling period should be between $(0.1772,0.3544) \mathrm{s}$, according to [23], i.e., $(28,56)$ tranmissions in a $10 \mathrm{~s}$ time, whereas the value for the minimum and maximum inter-event time are 0.081 and 2.478. Furthermore, this comparison is even unfair with the event-based approach, since once the system is around the equilibrium point, the broadcasting periods take values around 1-2 s.

Observe also that the control signals are piecewise constant (Figure 4c). They are updated if an event is triggered by the agent or its neighbors.

\subsection{Effect of the coupling terms}

Assume now a chain of inverted pendulums of length $N=200$ and that the matching condition does not hold, that is the terms $\Delta_{i j} \neq 0$ for $j \in$ $N_{i}$. Specifically, $\Delta_{i j} \in[-0.4,0.4]$ are generated, and these terms act as a disturbance to the system.

Figure 5 shows the output, the events generated and the control signal for the nodes 2, 3, 4, 75 and 180, respectively. A disturbance is induced at 

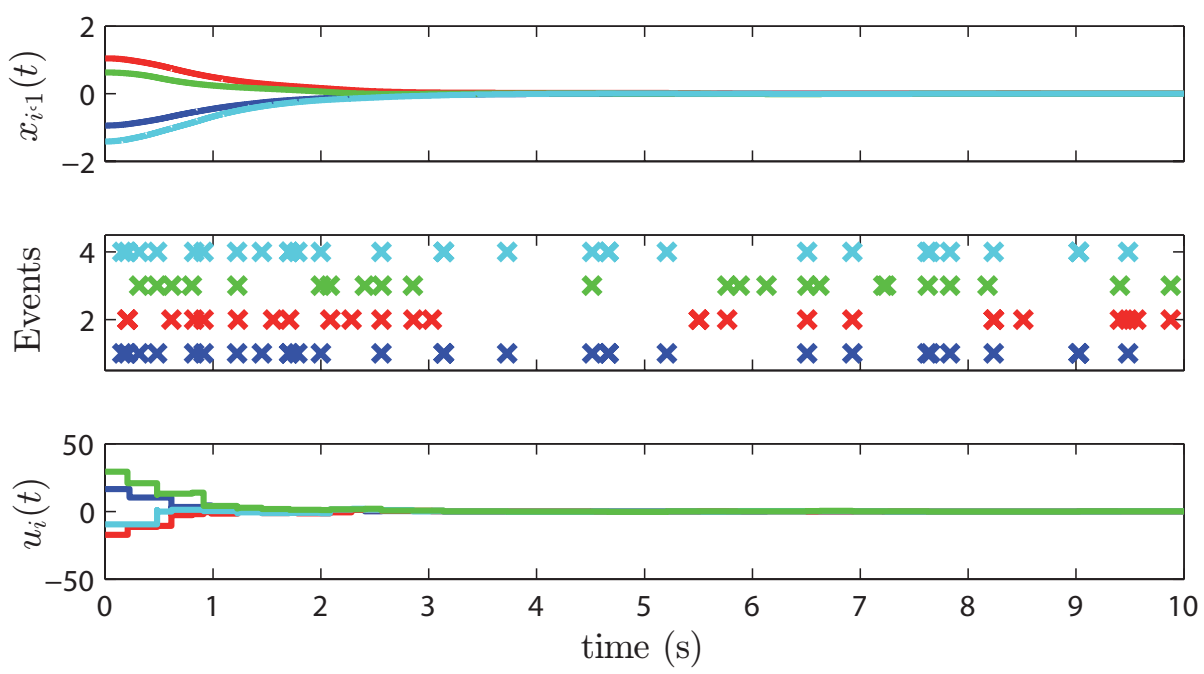

Figure 4: Simulation result with trigger functions (14), $c_{0}=0.02, c_{1}=$ 0.5 and $\alpha=0.7$

time $t=7 \mathrm{~s}$ at the pendulum 3 (blue line). Observe how the disturbance also affects the neighbors of the third node, 2 and 4 (red lines). However, there is no effect over nodes which are far away from the third one (green lines).

We can conclude that the event-based communication respects somehow the idea of neighborhood in a large scale system. Specifically, in a interconnected linear system, even if the system is not perfectly decoupled, the generation of events at a node takes place when something occurs (for instance, a disturbance), and an area around this node starts communicating in order to reject the disturbance, but the rest of the system is not affected.

\subsection{Model based control}

In Section 4 a model based approach was presented as a means of improving the event-based control of Section 3. Figure 6 compares the output of agent 1 when a simulation under the conditions described in Section 5.1 is performed. Observe that, for this case, the model-based approach reduces the number of events in more than a third (from 23 (in red) to 9 (in blue)). Note that the control law is not anymore a constant piecewise function.

Table 2 extends this study for a larger number of agents. Several simulations were performed for different initial conditions for each value of $N$. Mininum and mean values of the inter-event times $\left\{\tau_{k}^{i}\right\}$ were calculated for the set of the simulations with the same number of agents. We see that the 

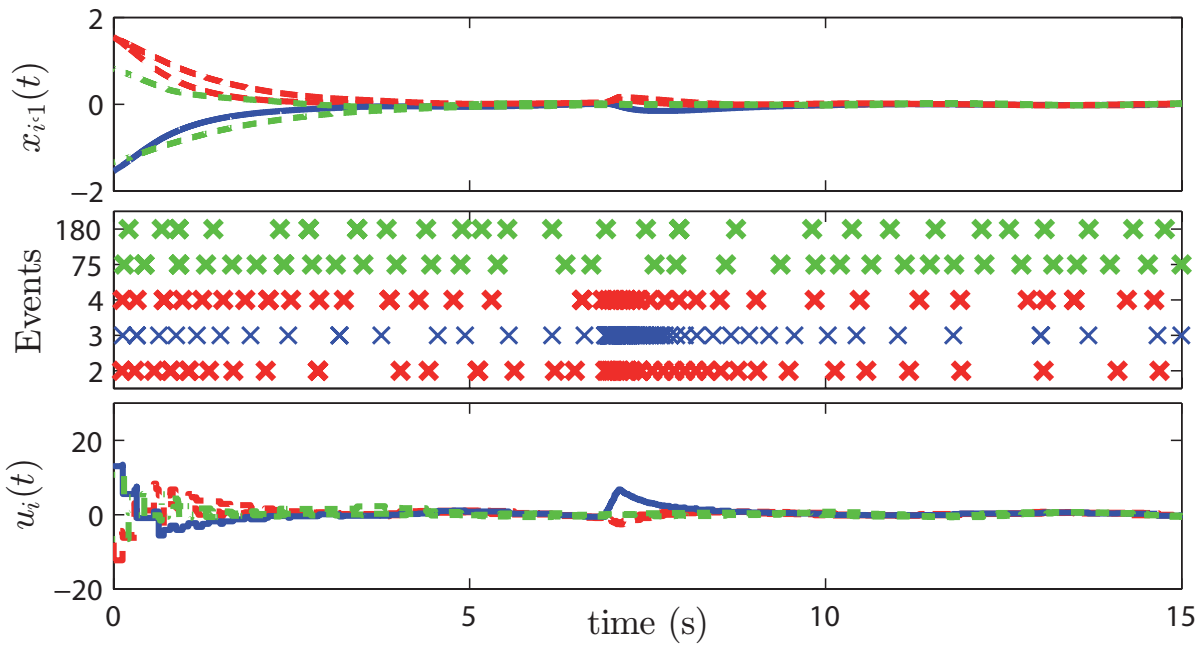

Figure 5: System response when $N=200$ when $\Delta_{i j} \in[-0.4,0.4]$
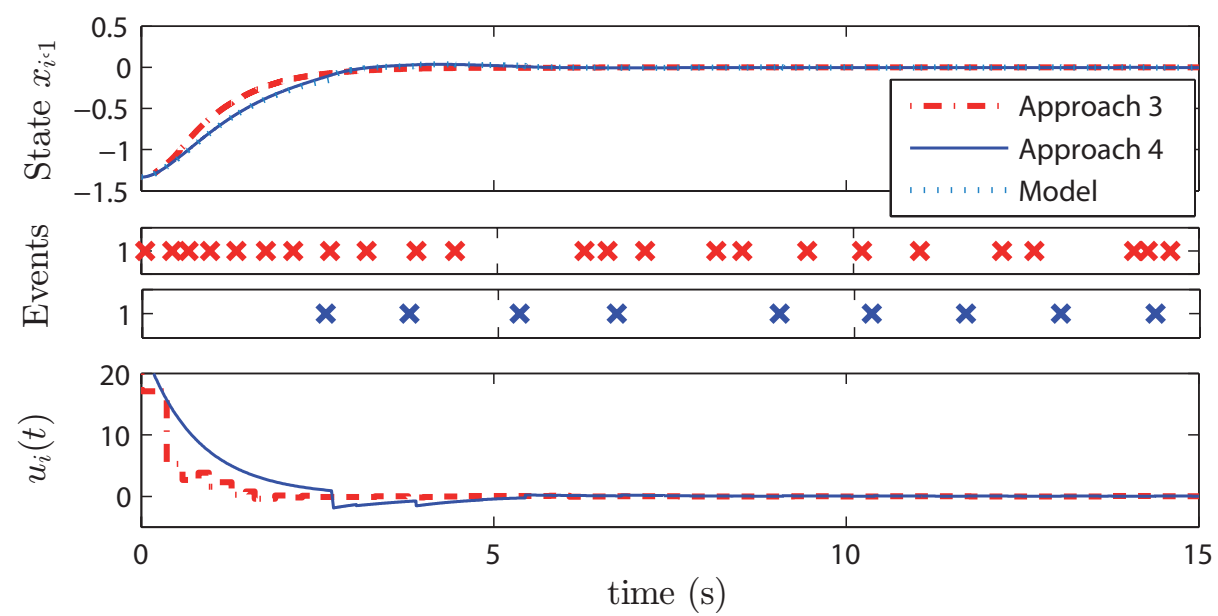

Figure 6: Simulation result with trigger functions (14) for the approaches of the sections 3 and 4 
Table 2: Inter-event times for different $N$

\begin{tabular}{|c|c|c|c|c|c|c|}
\hline Numl & er of agents & 10 & 50 & 100 & 150 & 200 \\
\hline Trigger condition & 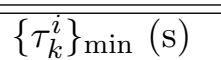 & 0.053 & 0.031 & 0.015 & 0.019 & 0.009 \\
\hline (14), Section 3 & $\left\{\tau_{k}^{i}\right\}_{\text {mean }}(\mathrm{s})$ & 0.565 & 0.565 & 0.567 & 0.572 & 0.568 \\
\hline Trigger condition & $\left\{\tau_{k}^{i}\right\}_{\min }(\mathrm{s})$ & 0.6816 & 0.3025 & 0.219 & 0.0963 & 0.132 \\
\hline (14), Section 4 & $\left\{\tau_{k}^{i}\right\}_{\text {mean }}(\mathrm{s})$ & 1.430 & 1.500 & 1.477 & 1.668 & 1.581 \\
\hline $\begin{array}{l}\text { Trigger condition } \\
\text { of }[14]\end{array}$ & $\left\{\tau_{k}^{i}\right\}_{\text {mean }}(\mathrm{s})$ & 0.1149 & 0.1175 & 0.1152 & 0.1180 & 0.1177 \\
\hline
\end{tabular}

model-based approach gives around three times larger broadcasting periods, remaining almost constant when the number of agents increases. Thus, the amount of communication for the overall network grows linearly with $N$.

Moreover, if we compare these results to [14, we see that the proposed scheme can provide around five times larger broadcast periods. For example, for a number of pendulums of $N=100$, trigger functions of the form (14) give mean broadcasting periods of 0.567 and 1.477 for the approaches described in sections 3 and 4, respectively, while the trigger functions in [14] give a mean value of 0.1152 . Though the scheme in [14] ensures asymptotic stability, we guarantee the convergence to an arbitrary small region around the origin with $c_{0} \neq 0$. Alternatively, one can choose $c_{0}=0$ to get rid of this drawback.

\section{Conclusions}

We presented a novel distributed event-based control strategy for linear interconnected subsystems. The events are generated by the agents based on local information only, broadcasting their state over the network. The proposed time-dependent trigger functions preserve the desired convergence properties and guarantee the existence of a strictly positive lower bound for the broadcast period, excluding the Zeno behavior.

A model-based approach is presented as a means of reducing the number of events. We prove that under certain conditions on the model uncertainty, the model-based approach gives larger inter-event times. The theoretical results and the contribution of the current paper with respect to previous work are illustrated through computer simulations.

The use of distributed algorithms [24, 25] in an event-based fashion to estimate global information of the system, in particular, $\lambda_{\max }\left(A_{K}\right)$ and $\|\Delta\|$, in order to establish bounds on the trigger function parameters, for instance $\alpha$, are possible directions of future research. 


\section{Appendix}

\section{Relaxing Assumption 1}

If Assumption 1 holds, then

$$
\left\|e^{A_{K} t}\right\|=\left\|V e^{D t} V^{-1}\right\| \leq\|V\| e^{-\left|\lambda_{\max }\left(A_{K}\right)\right| t}\left\|V^{-1}\right\|
$$

where $\lambda_{\max }\left(A_{K}\right)$ is the larger real part of the eigenvalues of $A_{K}$. Based on (44), the results of Theorem 3 are derived. We next bound $\left\|e^{A_{K} t}\right\|$ when $A_{K}$ is no longer diagonalizable and is decomposed into a general Jordan form.

Denote by $J$ the Jordan matrix so that $A_{K}=V J V^{-1}$. The matrix $J$ has $n$ Jordan blocks $J_{i}$, one for each eigenvalue $\lambda_{i}$. The dimension of each $J_{i}$ depends on the algebraic multiplicity of the associated eigenvalue $\lambda_{i}$, that is denoted by $q_{i}$. Thus, $J=\oplus_{i=1}^{n} J_{i}\left(\lambda_{i}\right)$ and hence, $e^{J}=\oplus_{i=1}^{n} e^{J_{i}\left(\lambda_{i}\right)}$.

From the properties of the block matrices it is strightforward that

$$
\left\|e^{J t}\right\| \leq \max _{i=1}^{n}\left(\left\|e^{J_{i}\left(\lambda_{i}\right) t}\right\|\right) .
$$

We next bound $\left\|e^{J_{i}\left(\lambda_{i}\right) t}\right\|$. Note that if Jordan blocks are diagonal then $\left\|e^{J_{i}\left(\lambda_{i}\right) t}\right\| \leq e^{\lambda_{i} t}$.

A Jordan block $J_{i}\left(\lambda_{i}\right)$ can be decomposed as

$$
J_{i}\left(\lambda_{i}\right)=\lambda_{i} I_{q_{i} \times q_{i}}+N_{i},
$$

where $N_{i}$ is a nilpotent matrix of the form

$$
N_{i}=\left(\begin{array}{ccccc}
0 & 1 & 0 & \ldots & 0 \\
0 & 0 & 1 & \ldots & 0 \\
\vdots & \vdots & & \ddots & \vdots \\
0 & 0 & \ldots & 0 & 1 \\
0 & \ldots & \ldots & \ldots & 0
\end{array}\right)
$$

This special nilpotent matrix has the property that $N_{i}^{k}=0, \forall k>=q_{i}$, being $q_{i}$ the algebraic multiplicity of $\lambda_{i}$ and so, the dimension of $N_{i}$. Thus, $e^{N_{i}}=$ $\sum_{k=0}^{q_{i}-1} \frac{N_{i}^{k}}{k !}$.

Note that in case of complex eigenvalues the term $\lambda_{i} I_{q_{i} \times q_{i}}$ in (46) is replaced by $C_{i} I_{q_{i} \times q_{i}}$, where $C_{i}$ are block matrix of the form

$$
C_{i}=\left(\begin{array}{cc}
a_{i} & b_{i} \\
-b_{i} & a_{i}
\end{array}\right)
$$

where $\lambda_{i}=a_{i}+j b_{i}$. Since $A_{K}$ is Hurwitz, $a_{i}<0$. 
Thus, if follows that

$$
\left\|e^{J_{i}\left(\lambda_{i}\right) t}\right\|=\left\|e^{C_{i} I_{q_{i} \times q_{i}} t} \sum_{k=0}^{q_{i}-1} \frac{N_{i}^{k} t^{k}}{k !}\right\| \leq\left\|e^{C_{i} I_{q_{i} \times q_{i}} t}\right\|\left\|\sum_{k=0}^{q_{i}-1} \frac{N_{i}^{k} t^{k}}{k !}\right\|,
$$

and since $\left\|N_{i}\right\|=1$, it yields

$$
\left\|e^{J_{i}\left(\lambda_{i}\right) t}\right\| \leq e^{-\left|a_{i}\right| t} \sum_{k=0}^{q_{i}-1} \frac{t^{k}}{k !} .
$$

Thus, if we denote by $\lambda_{\max }$ the real part of the eigenvalue $i$ for which its Jordan form meets (45), thus

$$
\left\|e^{A_{K} t}\right\| \leq\|V\|\left\|V^{-1}\right\| e^{-\left|\lambda_{\max }\right| t} \sum_{k=0}^{q_{\max }-1} \frac{t^{k}}{k !}
$$

where $q_{\max }$ is the algebraic multiplicity of the cited eigenvalue.

\section{References}

[1] Åstrom, K.J.: 'Event based control', in A. Astrolfi and L. Marconi (Ed.): 'Analysis and Design of Nonlinear Control Systems'. Springer Verlag, 2008.

[2] Tabuada, P.: 'Event-triggered real-time scheduling of stabilizing control tasks', IEEE Transactions on Automatic Control, 2007, 52(9), pp. 16801685 .

[3] Cervin, A., Henningsson, T.: 'Scheduling of event-triggered controllers on a shared network', 47th IEEE Conference on Decision and Control, 2008, pp. 3601-3606.

[4] Heemels, W.P.M.H., Sandee, J.H., and Van den Bosch, P.P.J.: 'Analysis of event-driven controllers for linear systems', International Journal of Control, 2008, 81(4), pp. 571-590.

[5] Rabi, M., Johansson, K.H.: 'Scheduling packets for event-triggered control', European Control Conference, Budapest, 2009, pp. 3779-3784.

[6] Lunze, J., Lehmann, D.: 'A state-feedback approach to event-based control', Automatica, 2010, 46(1), pp. 211-215. 
[7] Dimarogonas, D.V., Johansson, K.H.: Event-triggered control for multiagent systems, Shanghai, 48th IEEE Conference on Decision and Control, 2009, pp. 7131-7136.

[8] De Persis, C., Sailer, R., Wirth, F.: 'On a small-gain approach to distributed event-triggered control', 18th IFAC World Congress, Milano, 2011.

[9] Guinaldo, M., Dimarogonas, D.V., Johansson, K.H., Sánchez, J., Dormido, S.: 'Distributed Event-Based Control for Interconnected Linear Systems', 50th IEEE Conference on Decision and Control, Orlando, 2011, pp. 2553-2558.

[10] Mazo, M., Tabuada, P.: 'Decentralized event-triggered control over wireless sensor/actuator networks', IEEE Transactions of Automatic Control, 2011, 56(10), pp. 2456-2461.

[11] Molin, A., Hirche, S.: 'Optimal design of decentralized event-triggered controllers for large-scale systems with contention-based communication', 50th IEEE Conference on Decision and Control, Orlando, 2011, pp. 47104716.

[12] Seyboth, G.S., Dimarogonas, D.V., Johansson, K.H.: 'Control of MultiAgent Systems via Event-based Communication', 18th IFAC World Congres, Milano, 2011.

[13] Wang, X., Lemmon, M.D.: 'Event-triggering in distributed networked control systems', IEEE Transactions on Automatic Control, 2011, 56(3), pp 586-601.

[14] Wang, X., Lemmon, M.D.: 'Event-Triggered Broadcasting across Distributed Networked Control Systems', American Control Conference, Seattle, 2008, pp. 3139-3144.

[15] Seuret, A., and Prieur, C.: 'Event-triggered sampling algorithms based on a lyapunov function', 50th IEEE Conference on Decision and Control, Orlando, 2011.

[16] Wang, X., Lemmon, M.D.: 'Self-triggered feedback control systems with finite-gain L2 stability', IEEE Transactions on Automatic Control, 2009, 45(3), pp. 452-467.

[17] Anta, A., Tabuada, P.: 'To sample or not to sample: Self-triggered control for nonlinear systems', IEEE Transactions on Automatic Control, 2010, 55(9), pp. 2030-2042. 
[18] Montestruque, L.A., Antsaklis, P.J.: 'On the Model-Based Control of Networked Systems', Automatica, 2003, 39, pp. 1837-1843.

[19] Garcia, E., Antsaklis, P.J.: 'Decentralized Model-Based Event-Triggered Control of Networked Systems', American Control Conference, 2012, pp. 6485-6490. Longer version technical report found at http://www.nd.edu/ isis/techreports/isis-2012-002.pdf

[20] Zames, G., Bensoussan, D.: 'Multivariable Feedback, Sensitivity, and Decentralized Control', IEEE Transaction on Automatic Control, 1983, 28(11), pp. 1030-1035.

[21] Stefanovska, A.: 'Coupled Oscillators: Complex but Not Complicated Cardiovascular and Brain Interactions', IEEE Eng Med Biol Mag., 2007, 26(6), pp. 25-29.

[22] Diaz-Guilera, A., Arenas, A.: 'Phase Patterns of Coupled Oscillators with Application to Wireless Communication', in 'Bio-Inspired Computing and Communication', Springer-Verlag Berlin, 2008.

[23] Franklin, G.F., Powell, J.D., Workman, M.: 'Digital control of dynamic systems'. Addison-Wesley. E.U.A., 1997.

[24] Yang, F.P., Freeman, R.A., Gordon, G.J., Lynch, K.M., Srinivasa, S., Sukthankar, R.: 'Decentralized estimation and control of graph connectivity in mobile sensor networks', American Control Conference, Seattle, 2008.

[25] Yang, P., Freeman, R.A., Lynch, K.M.: 'Multi-agent coordination by decentralized estimation and control', IEEE Transactions on Automatic Control, 2008, 53(11), pp. 2480-2496. 